\title{
Formation of Dimers during the Initial Stage of Autoxidation in Methyl Linoleate
}

\author{
Kazuo Miyashita, Kenshiro Fujimoto and Takashi Kaneda \\ Department of Food Chemistry, Faculty of Agriculture, \\ Tohoku University, Sendai 980, Japan
}

Received August 10, 1981

\begin{abstract}
The formation of dimers in the initial stage of methyl linoleate (ML) autoxidation was demonstrated. The oxidation profile of freshly prepared ML was followed by TLC during autoxidation by aeration at $30^{\circ} \mathrm{C}$ for $192 \mathrm{hr}$. After $24 \mathrm{hr}$ of autoxidation, the peroxide value of ML was still 0.6, and two unknown polar spots appeared besides intact ML and methyl linoleate hydroperoxides (MLHPO). These two spots were identified as dimers by successive gel and high performance liquid chromatographic separations and by molecular weight determination. The ratio of dimers/MLHPO reached a maximum ( 0.74$)$ after $96 \mathrm{hr}$ of autoxidation. This result indicates that the formation of dimers in the initial stage of autoxidation was slightly less than that of MLHPO. The dimers were linked through $-\mathrm{C}-\mathrm{O}-\mathrm{O}-\mathrm{C}-$ bonds and contained hydroperoxy and/or carbonyl groups and conjugated dienes.
\end{abstract}

Many papers have been published on the autoxidation of unsaturated oils and it is generally accepted that autoxidation of oils involves a free radical mechanism. ${ }^{1 \sim 6)}$ It is also well known that in the course of autoxidation of lipids, the primary stable oxidation products are monomeric hydroperoxides and many secondary products, including such as volatile carbonyls and polymers, are formed by the decomposition of accumulated hydroperoxides. ${ }^{1 \sim 6)}$ Especially in the latter half of the autoxidation period, polymerization is the major reaction producing free radical termination. Consequently, the dimers or polymers thus far separated from autoxidized oils have been limited to the latter period of autoxidation. ${ }^{7 \text { (9) }}$ However, free radicals which are capable of forming dimers are present in oils even during the induction period of autoxidation. Therefore, it can be assumed that a part of these radicals may polymerize to form dimers in an early stage of autoxidation. However, nobody has reported the formation of dimers in this stage. In this paper, dimers were separated from autoxidized ML during the initial stages of oxidation by some chromatographic techniques, and the structure of linkages were discussed.

\section{MATERIALS AND METHODS}

Preparation of $M L$. ML was prepared from mixed methyl esters of safflower oil fatty acids by the method of urea adduction ${ }^{10)}$ and purified of a trace amount of impurities such as hydroperoxides and tocopherols by reduced pressure distillation and silicic acid column chromatography. This was done just before its use.

Oxidation procedure. Approximately $150 \mathrm{~g}$ of ML was autoxidized at $30 \pm 2^{\circ} \mathrm{C}$ by bubbling with dry air for $192 \mathrm{hr}$. Samples aliquots were taken to analyze the peroxide value (POV, AOCS official method) and thin layer chromatographic patterns. Thin layer chromatography was carried out using Kieselgel 60 (precoated: $0.25 \mathrm{~mm}$ thick, Merck) plates and developed with a solvent system consisting of hexane-diethyl ether $(70: 30)$. Spots were visualized by spraying with $50 \%$ aqueous sulfuric acid and subsequent charring at $110^{\circ} \mathrm{C}$ for $10 \mathrm{~min}$. For quantitative analysis, the charred chromatograms were scanned on a Shimadzu Densitometer CS-900 using the transmittance mode with zig-zag scanning at $450 \mathrm{~nm}$, and recorded on a Shimadzu Recorder U-225 MCS.

Isolation of dimers from autoxidized $M L$. The autoxidized ML was fractionated by gel chromatography on a Bio-Beads S-X3 (Bio-Rad) column $(180 \times 2.7 \mathrm{~cm})$. Approximately $1.5 \mathrm{~g}$ of oxidized ML $(120 \mathrm{hr}$ : POV $=16)$ was separated with benzene (flow rate, $0.7 \mathrm{ml} / \mathrm{min}$ ) and 
$8 \mathrm{ml}$ fractions were collected. After the removal of benzene in vacuo and successive dissolving in methanol $(5 \mathrm{ml})$, the amounts of eluted oxidized esters was assayed by UV spectrometry with the effluent at $233 \mathrm{~nm}$ for conjugated diene functionality. The dimeric fraction (Fr.-1 in Fig. 3) obtained by gel chromatography was further separated by high performance liquid chromatography (HPLC). HPLC was carried out on a $40 \times 0.8 \mathrm{~cm}$ pyrex column packed with Lichrosorb RP-18 (Merck) at $1.9 \mathrm{ml} / \mathrm{min}$ flow rate with a Seishin Model S-155D pumping system (Seishin Pharmaceutical Co., Tokyo). The column eluent was monitored with a variable wavelength UV detector (Uvidec-100-III, JASCO Co., Tokyo) set at $233 \mathrm{~nm}$. The column was eluted with a mixture of acetonitrile--watertetrahydrofuran $(15: 4: 1)$.

Analytical procedures. Mean molecular weights (MMW) of chromatographic fractions were determined by the vapor pressure equilibrium method using benzene on a Hitachi Perkin-Elmer Model 115 apparatus. For the detection of hydroperoxy groups in the oxidized products on TLC, a $1 \% N, N^{\prime}$-dimethyl-p-phenylenediamine (DMPD) solution consisting of the mixture of methanol-wateracetic acid $(128: 25: 1)$ was employed. ${ }^{11)}$ To characterize the intermolecular linkages of the dimers, first, the dimeric fraction was treated with sodium borohydride ${ }^{12)}$ and reduced preparations were analyzed by gel chromatography under exactly the same conditions as described above.

\section{RESULTS AND DISCUSSION}

A typical series of densitograms of autoxidized ML at various oxidation stages are shown in Fig. 1. Judging from the POV, the induction period for autoxidation under this condition was approximately $72 \mathrm{hr}$ (Fig. 2). At the initiation of oxidation, only a single peak derived from the intact ML with an $R f$ value of 0.5 was recognized. However, after $24 \mathrm{hr}$, POV was still 0.6, and three new peaks, $\mathrm{A}_{1}(R f=$ $0 \sim 0.1), \mathrm{A}_{2}(R f=0.1 \sim 0.2)$ and $\mathrm{A}_{3}(R f=0.22)$, appeared besides the ML. Both $A_{1}$ and $A_{2}$ were assumed to be mixtures of several components because they formed rather wide bands. These peaks were completely absent on the TLC before oxidation. Among these unknown peaks, $A_{3}$ was identified as MLHPO by comparison with the authentic specimen on TLC and HPLC. ${ }^{13)}$ The variations in the percentage distribution of these components $\left(\mathrm{A}_{1}\right.$, $\left.\mathrm{A}_{2}, \mathrm{MLHPO}, \mathrm{ML}\right)$ during autoxidation were estimated by a densitometer and are shown in Fig. 2. After $24 \mathrm{hr}$, the amount of $A_{1}$ did not increase but decreased slightly $(0.1 \sim 0.2 \%)$ with the proceeding of oxidation. On the other hand, $\mathrm{A}_{2}$ increased rapidly after 72 to $96 \mathrm{hr}$, but then remained unchanged thereafter. The ratio of $A_{1}+A_{2} /$ MLHPO reached a maximum (0.74) after $96 \mathrm{hr}$, in which POV was 10.7. Therefore, the total amount of these unknown polar substances $\left(\mathrm{A}_{1}, \mathrm{~A}_{2}\right)$ was slightly less than that of MLHPO in the early stages of autoxi-
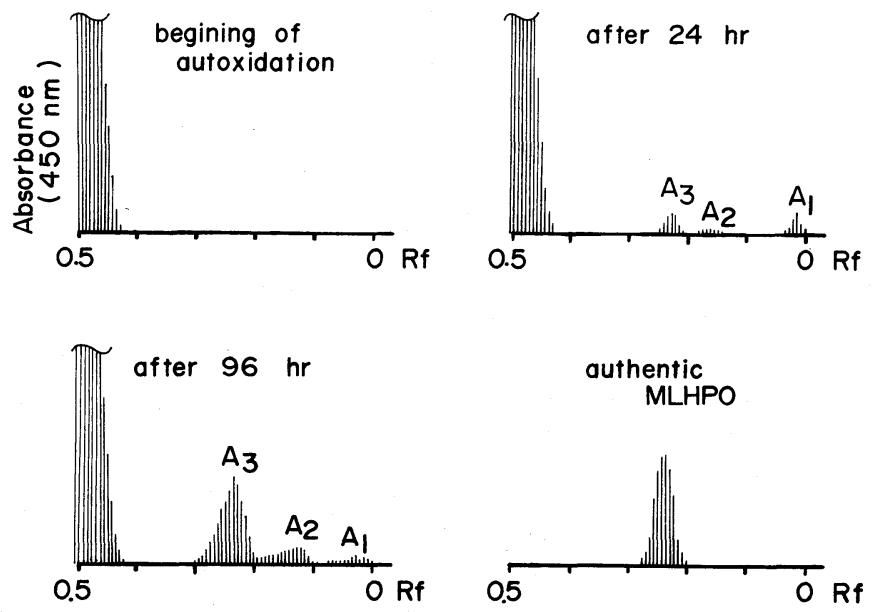

FIG. 1. Densitograms of Autoxidized ML.

Samples were developed on Kieselgel $60(0.25 \mathrm{~mm})$ with $n$-hexane-diethyl ether $(70: 30, \mathrm{v} / \mathrm{v})$. The spots were detected by spraying with $50 \%$ aqueous sulfuric acid, followed by heating for $10 \mathrm{~min}$ at $110^{\circ} \mathrm{C}$. Thin layer chromatograms were scanned with a zig-zag scanning densitometer (Shimadzu CS-900). 


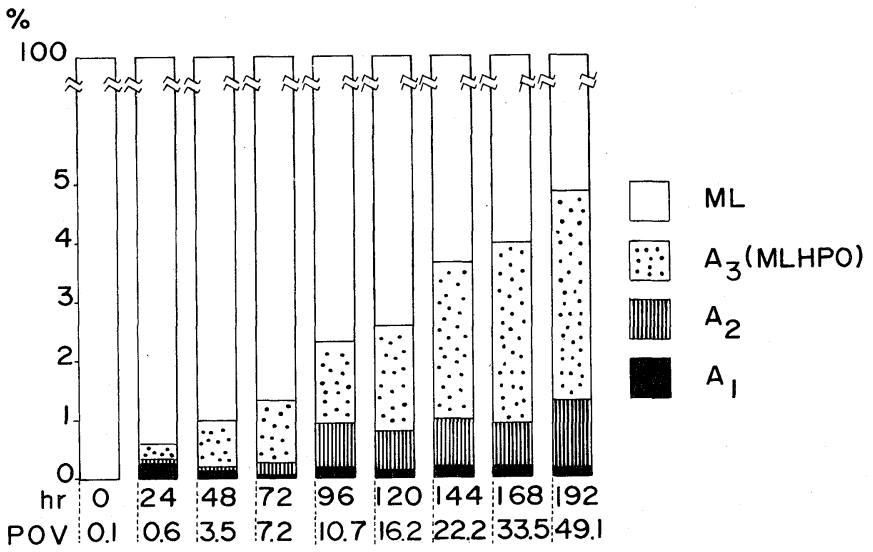

FIG. 2. Quantitative Analysis of Autoxidized ML by Photodensitometer.

Percentage distribution of each component $\left(A_{1}, A_{2}, A_{3}, M L\right)$ was calculated from the peak area on densitograms. TLC was carried out under the same conditions as in Fig. 1.

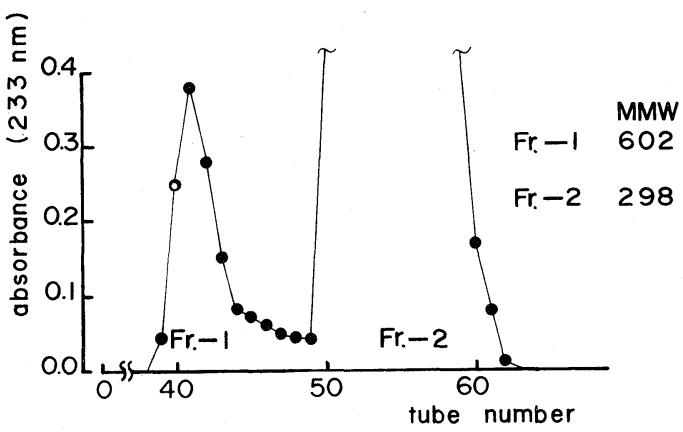

FIG. 3. Fractionation of Autoxidized ML (POV: 16.2) on a Bio-Beads S-X3 Column.

Autoxidized ML (1.5 g) was eluted on the column of BioBeads S-X3 $(2.7 \times 180 \mathrm{~cm})$ with benzene at flow rate of $0.7 \mathrm{ml} / \mathrm{min}$, and $8 \mathrm{ml}$ fractions were collected. After removal of the benzene and addition of $5 \mathrm{ml} \mathrm{MeOH}$, optical density of each fraction was measured at $233 \mathrm{~nm}$. MMW of Fr.-1 and Fr.-2 was determined by the vaporpressure equilibrium method.

dation. On the assumption that these polar materials were dimers or polymers, the isolation of $\mathrm{A}_{1}$ and $\mathrm{A}_{2}$ from the autoxidized $\mathrm{ML}$ was attempted. Several conventional methods such as molecular distillation, ${ }^{14,15)}$ solvent extraction, ${ }^{8,16)}$ and column chromatography ${ }^{17)}$ have been applied for the separation of polymers from autoxidized or thermally oxidized oils, but in recent years, gel permeation chromatography has been successfully introduced for this process. ${ }^{18 \sim 21)}$ So we utilized a column

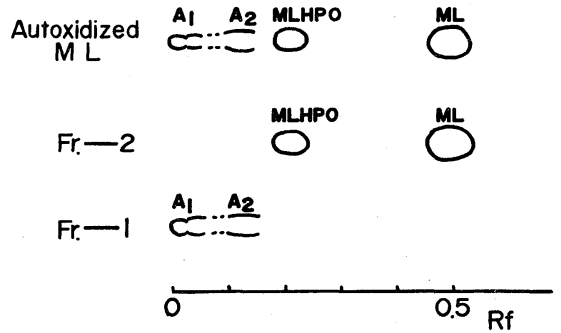

FIG. 4. Comparison of Fr.-1,2, and Autoxidized ML. TLC was carried out under the same conditions as in Fig. 1.

of polystyrene gel (Bio-Beads S-X3) for the separation of dimers or polymers. Via gel chromatography, the autoxidized ester was separated into two fractions (Fig. 3). After evaporation of the solvent, the molecular weight of both fractions was determined. As a result, the MMW of Fr.-1 was found to be 602 . The elution volume for Fr.-1 on this column was in fair agreement with that for authentic glycerol trilaurate (molecular weight, 639; Fr. 39 42) whose molecular weight is almost equal to those of dimers of ML. These combined results suggested that this fraction is principally composed of dimers. On the other hand, the MMW of Fr.-2 agreed with the monomer of ML (found, 298; theoretical value for ML, 294). By comparing Fr.-1 and 2 with the unfractionated autoxidized $\mathrm{ML}(\mathrm{POV}=$ 
16) on TLC (Fig. 4), it was revealed that both $A_{1}$ and $A_{2}$ were satisfactorily isolated from intact ML and MLHPO, and found only in the dimer fraction (Fr.-1). Furthermore, $A_{1}$ and $A_{2}$ showed different retention times on reverse phase $\operatorname{HPLC}\left(\mathrm{A}_{1}, 3.5 \sim\right.$ $4.4 \mathrm{~min} ; \mathrm{A}_{2}, 6.0 \sim 7.5 \mathrm{~min}$ ), which has enabled the isolation of $A_{1}$ and $A_{2}$ from Fr.-1. The measurement of $\mathrm{MMW}$ of $\mathrm{A}_{1}$ and $\mathrm{A}_{2}$ prepared in this way $\left(A_{1}, 660 ; A_{2}, 619\right)$ confirmed that both of these components were dimers. HPLC also showed that both $\mathrm{A}_{1}$ and $\mathrm{A}_{2}$ were not made of single compounds, but composed of several overlapped peaks, suggesting the complexity of dimers. Therefore, it was ascertained that a considerable amount of dimers other than MLHPO were formed in a very early stage of autoxidation, in contrast with the conventional scheme of oxidation in which dimer formation occurred in a latter stage of autoxidation. ${ }^{2,4,5)}$

Figure 5 represents TLC of Fr.-1 and 2

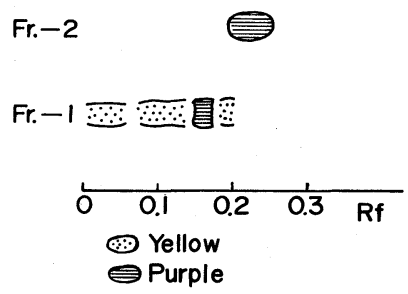

FIG. 5. TLC of Fr.-1,2 on Kieselgel 60 with $n$-HexaneDiethyl Ether $(70: 30, \mathrm{v} / \mathrm{v})$.

Visualization of spots was carried out by spraying the plate with $N, N^{\prime}$-dimethyl-p-phenylenediamine. visualized with DMPD reagent. In Fr.-1, a distinct purple spot $(R f=0.17)$ and three yellow spots $(R f=0.21,0.13,0.07)$ were detected. On the other hand, Fr.-2 produced one purple spot corresponding to MLHPO. As it is known that the reaction of DMPD with organic hydroperoxides like MLHPO results in a purple coloration, it is suggested that the main component of dimers in $\mathrm{A}_{2}$ consists of one or more hydroperoxy groups. While the principle responsible for yellow coloration with DMPD has not been throughly elucidated, Benedetti et $a .^{22)}$ pointed out that the fractions stained yellow by DMPD in dialyzable products originating from the peroxidation of liver microsomal lipids contained most of the carbonyl functional groups detectable in the dialyzate. Therefore, the presence of carbonyl groups in the dimer fraction was suggested. These observations were confirmed via IR spectra which showed the presence of carbonyls by absorption at $1700 \sim 1730 \mathrm{~cm}^{-1}$. Furthermore, IR and UV analyses indicated that the dimers contained a conjugated diene system.

The change of chromatographic patterns of Bio-Beads columns of the dimer fraction (Fr.1) after reduction with sodium borohydride is shown in Fig. 6. Following depolymerization with the reductant, more than $90 \%$ of the reduced products eluted in the same position as the monomer (compare with Fig. 3). This result suggested that most dimers were linked through $-\mathrm{C}-\mathrm{O}-\mathrm{O}-\mathrm{C}-$ bonds, because other possible linkages, such as $-\mathrm{C}-\mathrm{C}-$ and $-\mathrm{C}-\mathrm{O}-$

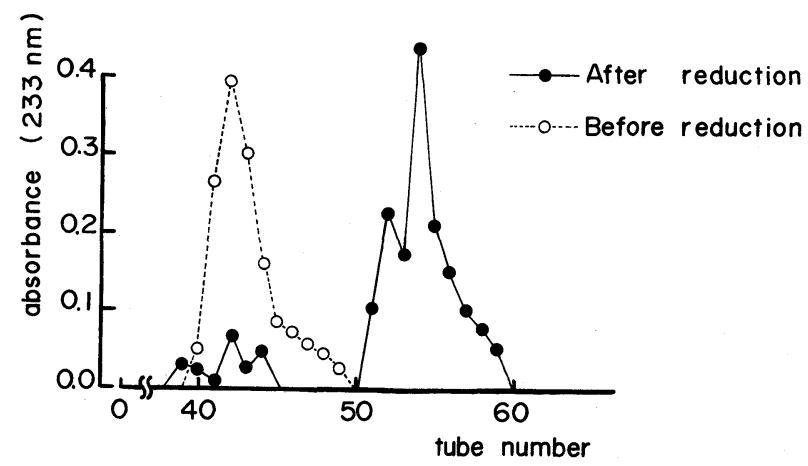

FIG. 6. Elution Patterns of Reduced Products Derived from Original Dimer Fraction. 
C-, are not severed by reduction with sodium borohydride.

Concerning the structure of oxidative polymers derived from unsaturated esters, Chang and $\mathrm{Kummerow}^{8)}$ have isolated dimer and trimer fractions by solvent extraction from ethyl linoleate autoxidized by aeration with dry air at $30^{\circ} \mathrm{C}$ for $350 \mathrm{hr}$. They concluded the esters were polymerized through $-\mathrm{C}-\mathrm{O}-\mathrm{O}-\mathrm{C}-$ bonds. Compared to their experimental conditions, the sample ester we employed was in an earlier stage of autoxidation. However, the basic structure of the dimers seemed to be identical. In the case of thermal decomposition of hydroperoxides and thermal oxidation of oils, dimers were linked through $-\mathrm{C}-\mathrm{C}-$ bonds. ${ }^{15,23,24)}$ Therefore, the dimers linked through $-\mathrm{C}-\mathrm{O}-\mathrm{O}-\mathrm{C}-$ bonds should be characteristic of autoxidized oils.

\section{REFERENCES}

1) E. H. Farmer, G. F. Bloomfield, A. Sundralingam and P. A. Sutton, Trans. Faraday Soc., 38, 348 (1942).

2) R. T. Holman, "Progress in the Chemistry of Fats and Other Lipids," Vol. II, Pergamon Press Ltd., London, 1954, p. 51.

3) W. O. Lundberg, "Autoxidation and Antioxidants," Vol. I, Interscience Publ. Co., New York, N. Y., 1961, p. 55.

4) C. H. Lea, "Symposium on Foods, Lipids and their Oxidation," Avi Publ. Co., Westport, Connecticut, 1962, p. 3.

5) R. Paoletti and D. Kritchevsky, "Advances in Lipids
Research," Vol. VII, Academic Press Ltd., New York, N. Y., 1969, p. 245.

6) E. N. Frankel, "Prog. Lipid Res.," Vol. XIX, Pergamon Press Ltd., London, 1980, p. 1.

7) S. S. Chang and F. A. Kummerow, J. Am. Oil Chem. Soc., 30, 251 (1953).

8) S. S. Chang and F. A. Kummerow, J. Am. Oil Chem. Soc., 30, 403 (1953).

9) D. Swern et al., J. Am. Chem. Soc., 67, 1132 (1945).

10) J. G. Keppler, S. Sparreboom, J. B. A. Stroink and J. D. von Mikusch, J. Am. Oil Chem. Soc., 36, 308 (1959).

11) J. G. Kirchner, "Technique of Organic Chemistry," Vol. VII, Wiley-Interscience, New York, N. Y., 1967, p. 159.

12) T. Ohfuji and T. Kaneda, Lipids, 8, 353 (1973).

13) H. W.-A. Chan and G. Levett, Lipids, 12, 99 (1977).

14) E. G. Perkins and F. A. Kummerow, J. Am. Oil Chem. Soc., 36, 371 (1959).

15) E. N. Frankel, C. D. Evans and J. C. Cowan, J.Am. Oil Chem. Soc., 37, 418 (1960).

16) L. A. Witting, S. S. Chang and F. A. Kummerow, J. Am. Oil Chem. Soc., 34, 470 (1957).

17) E. N. Frankel, C. D. Evans, H. A. Moser, D. G. MaConnell and J. C. Cowan, J. Am. Oil Chem. Soc., 38, 130 (1961).

18) H. Inoue, K. Konishi and N. Taniguchi, $J$. Chromatogr., 47, 348 (1970).

19) K. Aitzetmuller, J. Chromatogr., 71, 355 (1972).

20) E. G. Perkins, R. Travboid and A. Hsieh, J. Am. Oil Chem. Soc., 50, 223 (1973).

21) J. Pokorny, M. K. Kundu, S. Pokorny, M. Bleha and J. Coupek, Die Nahrung, 20(2), 157 (1976).

22) A. Benedetti, A. F. Casini, M. Ferrali and M. Comporti, Biochem. Pharmacol., 28, 2909 (1979).

23) L. Williamson, J. Appl. Chem., 3, 301 (1953).

24) M. M. Paulose and S. S. Chang, J. Am. Oil Chem. Soc., 50, 147 (1973). 